\title{
Similarities of low-dimensional chaotic auditory attractor sequences to quasirandom noise
}

\author{
ROBERT A. M. GREGSON and JOHN P. HARVEY \\ Australian National University, Canberra, Australian Capital Territory, Australia
}

\begin{abstract}
The production of sequences of sounds of various pitch levels from the algebra of chaotic attractors' trajectories is relatively straightforward. Meyer-Kress (cited in Kaneko, 1986) suggested that such sequences would be distinguishable from random independent identically distributed sequences. In psychophysical terms, this is a pattern-discrimination or pattern-similarity perception task, but these two tasks are not exactly the same thing. Nine attractors from the algebras of Henon, Zaslavskii (1978), Kaplan and Yorke (1979), Lorenz, and Gregson, and the logistic and Baker transformations, were paired with 10 realizations of a random series. The identification of the random member in each pair, the confidence of identification, and the perceived pairwise similarity were recorded by 65 subjects without initial feedback and by 76 subjects with initial feedback on five trials only, for each of 20 such pairs. The results indicate varying degress of discriminability; they can be expresssed in an analog of the receiver-operating characteristics of the attractors. There is no evidence of any homogeneous basis for the discrimination, and subjects who perform better are apparently not using the same bases as those who perform poorly. The fractal dimensionality of attractors may furnish a basis for their recognition, or the consequent autoregressive spectra induced in finite (short) samples, but recent work suggests the latter spectra can be insensitive to low-dimensional attractor dynamics.
\end{abstract}

A dynamic process is by definition something that evolves through time, and its mathematical representation is characterized both by the variables that themselves evolve, creating a time series of realizations of the process (either continuously or in discrete steps), and by parameters that determine the form of the evolution. The values of these parameters are critical, because in a nonlinear process, very small changes in the parameters can induce vast changes in the evolutionary dynamics. There is no necessary proportionality between changes in the values of the parameters governing the evolution of the process in time and the changes in the evolutionary path itself. This evolution of the process is sometimes called the trajectory of the variables.

In some circumstances, which can be specified completely by the parameter values and the starting values of the process variables, the trajectory locks into a stable pattern, which is called an attractor. An attractor can be a single point, a cycle of fixed periodicity in the time units of the trajectory, or can take one of various more complicated forms, some of which are called chaotic. Chaotic behavior resembles random behavior but is completely deterministic. It is not locally predictable beyond a very short time ahead, and the degree of its unpredictability is

The work was supported by Australian Research Council Grant A78831403 to the first author in 1990 . The text was originally prepared for a paper at the 17th Australian Experimental Psychology Conference, Australian National University, Canberra, A.C.T. in July 1990. We are indebted to K. Briggs, Mathematics Department, La Trobe University, Melbourne, Australia, for comments. Requests for reprints should be sent to R. A. M. Gregson, Psychology Department, The Faculties, Australian National University, G.P.O. Box 4, Canberra, A.C.T. 2601, Australia quantifiable from properties of its algebra. The Lyapunov exponents are the most usual way to define the dynamics in this sense.

The Lyapunov exponents measure the rate at which nearby trajectories diverge; "nearby" implies that they start close but are generated by slightly different parameter values that govern the evolution of the process in time. The largest positive Lyapunov exponent is most important since it dominates, in the direction associated with the divergence, the evolution of the attractor. The sum of the positive Lyapunov exponents of an attractor is called the Kolmogorov entropy, or K-entropy for short. This is a measure of the rate at which information is created by the dynamics of the system; in other words, it is the uncertainty that is resolved by taking further observations after the present. The patterns of signs of the Lyapunov exponents, of which there is one for each embedding dimension, are an indication of the type of dynamics present; for example, if one exponent is zero, and another is greater than zero, the presence of a folded sheet attractor is indicated.

There are often practical problems in estimating the parameter values that sufficiently describe an attractor's dynamics. An attractor is a pattern of activity embedded in an $m$-dimensional space; the dimensionality of this attractor can be measured in a number of different ways and need not be integer. The most common measure of the dimensionality is called the Hausdorff, or correlation, dimension, $D_{2}$; it is usually less than $m$. $D_{0}$ is the fractal dimension, and $D_{1}$ is the information dimension. The formal definitions of these measures are discussed, for example, by Feder (1988). $D_{0}$ is 0 for fixed points, 1 for 
limit cycles, and 2 for a two-torus. $D_{1}$ is always less than or equal to $D_{2}$.

The Hausdorff dimensionality of data is computationally difficult to estimate from fairly short time series, and the Kaplan and Yorke (1979) approximation, $D_{K Y}$, is more tractable.

As used by Briggs (1990), this dimensionality is defined as satisfying the following conditions: given $\boldsymbol{n}$ Lyapunov coefficients, $L_{1}, \ldots L_{\mathrm{i}}, \ldots L_{\mathrm{n}}$, which we can estimate from an algorithm of Wolf, Swift, Swinney, and Vastano (1985),

$$
D_{K Y}=k+\left(\sum_{i=1}^{k} L_{i}\right)|| L_{k+1} \mid,
$$

where $k$ is the minimum integer that satisfies

$$
\sum_{i=1}^{k} L_{i}>0, \text { and } \sum_{i=k+1}^{n} L_{i}<0 .
$$

If all $L_{i} \leq 0$, then we have a quasiperiodic process.

It is the case that chaos appears if and only if

$$
\max \left(L_{i}\right)>0 \text {, }
$$

there exists an $i$ such that $L_{i}=0$, and

\section{$D_{K Y}$ is noninteger.}

The mathematical properties that formally define attractors, ${ }^{1}$ as given above, are not necessarily those that the brain could somehow utilize to recognize chaos, when presented with a finite realization of the attractors' trajectories as time series, encoded in some sensory modality. It is conjectured (Meyer-Kress, cited in Kaneko, 1986) that we should be able to tell chaotic series from random ones, because, in a diversity of ways, the patterns generated by chaos are qualitatively different from completely unpredictable series in which all output values are equally probable, such as the $n$th order transition probabilities. Chaotic series are often locally recursive or quasiperiodic; we can compute descriptive statistics of a relatively very simple nature and use them as indicators of some, but not all, attractors. The members of the collection used here were chosen because they vary in their dynamics; they are all able to generate chaos in the mathematical sense of having appropriate patterns of Lyapunov coefficients, but with respect to their simple autoregressive properties, they are heterogenous. Thus, they present a wide exploratory family with which to test the conjecture that chaos is recognizable as nonrandomness to the untrained observer. The series generated here are not familiar, like melodies; it has been remarked by some observers that they resemble what has come to be called minimalist music, and it is obvious from inspection of their realizations that some would be easily discriminated from noise and others would not. However, discriminability is not of itself informative about the degree of perceived similarity between two series; it is obviously necessary for two things to have some difference if they are not to be located on the identity pole of the similarity continuum as used here and in many previous studies (Gregson, 1975), but determining that they are different tells us almost nothing about how different, in degree, they are perceived to be. In this sense, the conjecture of Meyer-Kress (cited in Kaneko, 1986) is not psychophysically adequate; more information is potentially in the situation than could be revealed simply by testing pairwise discriminability.

\section{Attractor Properties}

The problem of human discriminatory responses to stimuli with nonlinear and quasiperiodic dynamics is interesting because little is known about it and because its study brings together similarity psychometrics, time series analysis, and chaotic processes in psychophysics (Gregson, $1975,1983,1988)$.

Harvey (1990) created time series from a range of attractors whose mathematics are known and paired each of them with series generated by a random-sequence generator. The random sequences used in computer software are not strictly random but are themselves chaotic, since the ways in which they are generated produce attractors of integer or very high and possibly incommensurate dimensionality. They do, however, differ in their properties from some chaotic attractors that typically have low and fractal dimensionality, such as the examples considered here. Details of their algebraic forms are given below. The most comprehensive catalogue of attractor forms is that provided by Gumowski and Mira (1980). This set was chosen for its diversity and is not in any sense exhaustive in reproducing the possible properties of various types of chaos.

1. The Henon map, with $a=1.2$, is

$$
\begin{aligned}
& x_{n+1}=y_{n}+1-a x_{n}^{2} \\
& y_{n+1}=b x_{n}, x_{0}=0.1, y_{0}=0.1, b=0.3 .
\end{aligned}
$$

2. The Lorenz equations are

$$
\begin{aligned}
& d x / d t=-\sigma x+\sigma y \\
& d y / d t=-x z+r x-y \\
& d z / d t=x y-b z,
\end{aligned}
$$

with $r=28, \sigma=10, b=8 / 3, x_{0}=0, y_{0}=20, z_{0}=0$.

3. The Kaplan and Yorke (1979) equations are

$$
\begin{aligned}
& x_{n+1}=2 x_{n}(\bmod 1) \\
& y_{n+1}=\alpha y_{n}+\cos \left(4 \pi x_{n}\right),
\end{aligned}
$$

with $\alpha=0.2, x_{0}=0.35467895, y_{0}=0.1$.

4. The logistic pattern, creating a simple recursion, is

$x_{n+1}=a x_{n}\left(1-x_{n}\right), a=3.9847476, x_{0}=0.3$.

5. The Baker transformation is

$$
x_{n+1}=2 x_{n}(\bmod 1), \quad x_{0}=0.35467895 \text {. }
$$

6. The logistic map equation generating chaos is

$$
x_{n+1}=a x_{n}\left(1-x_{n}\right), \quad a=4.0, \quad x_{0}=0.3 \text {. }
$$


7. Zaslavskii's (1978) equation is

$$
\begin{aligned}
x_{n+1} & =\left[x n+v\left(1+\mu y_{n}\right)+\epsilon v \mu \cos 2 \pi x_{n}\right](\bmod 1) \\
y_{n+1} & =e^{-r}\left(y_{n}+\epsilon \cos 2 \pi x_{n}\right),
\end{aligned}
$$

with $v=400 / 3, \epsilon=0.3, \mu=\left(1-e^{-\Gamma}\right) / \Gamma, e=2.71828183$, $\Gamma=3.0, x_{0}=0.2, y_{0}=0.01$.

8. The Henon map, with $a=1.4$; otherwise as for (3).

9. The $\Gamma$ recursion (Campbell \& Gregson, 1990; Gregson, 1988, 1989a, 1989b; Gregson \& Britton, 1989; Price \& Gregson, 1988) in two-variable form is

$$
\begin{aligned}
x_{k+1} & =a\left(e^{2}-e^{2} x_{k}+x_{k}^{2}-y_{k}^{2}+3 x_{k} y_{k}^{2}-x_{k}^{3}\right) \\
y_{k+1} & =a y_{k}\left(-e 2+2 x_{k}-3 x_{k}^{2}+y_{k}^{2}\right), \\
\text { with }\left(x_{0}, y_{0}\right) & =(0.5, \epsilon), \epsilon \ngtr .2, a=6, e=0 .
\end{aligned}
$$

There are a number of $a, e$ settings that will yield chaotic behavior, because the recursion exhibits the Devil's Staircase (Mandelbrot, 1977).

Table 1 summarizes some descriptive parameters of the chaotic series that have been published up to the time of this analysis. It is incomplete because not all parameters have been determined. Where two values are given, more than one published determination exists. Frequently, the determination of such parameters is necessarily approximate and not analytic, requiring heavy computations on very long series that are thousands of times longer than those that could be used in a psychophysical experiment with human observers. The most important distinction in Table 1 is the difference in dimensionality between the attractors and random noise.

\section{Time Series Encoding}

The realizations of the attractors are time series, and every time series has an infinite-order autoregressive representation, derived from a well-known theorem of Parzen. In the present context, by using methods that are derived from Box and Jenkins (1970), it is sufficient to get an approximate representation in order to characterize the structure of a finite and relatively short sample.

It may be remarked that the human observer's information processing capacity is obviously not such that an infinite-order autoregression can be processed in short- term memory, and fitting an autoregressive moving average (ARMA) model with lag orders not greater than 3 is a priori about a plausible limit on representations of the observer's capacity to identify, without practice, short recurrent sequences in the stimulus series. Each of the attractors can thus be characterized by its autoregressive spectrum and by a low-order ARMA model that picks up only the pseudostationarities in the sample length. The intrinsic statistical problem with this approach is that the chaotic series behave as very nonstationary when an AR(I)MA (that is, integrated with differencing) representation is attempted, even with a difference order of 2 . As is usual with nonlinear processes, trying to get a linear model to fit induces some long lag components in the AR(I)MA form that are probably pseudoautocorrelations and not a function of the attractor $D_{2}$ dimensionality. ${ }^{2}$

\section{Discriminability and Similarity}

The first attempt to set up a model for the similarity of sequential patterns was made by Imai (cited in Gregson, 1975, section 6.34), who suggested that for short series, of 12 symbols, information processing is possibly performed through attempts to transform one pattern into another-the resulting similarity judgment is then inversely proportional to the effort required to make the transformation. This is a nice idea, but, for series as long as those used in the present study, it is an oversimplification; the similarities must rest on intermediate constructs that encode the long series in a terser form before comparisons between series are made.

Various such properties suggest themselves; the local autoregressions are one example because they are immediately related to the internal redundancy of the series. Attractors can show what is called intermittency, with short, not quite identically repeating, patterns embedded in unpredictable series. In turn, the local redundancy is related to the Lyapunov exponents; thus, we can find a way back from the psychophysics to the attractor measures.

\section{Neuropsychological Implications}

\begin{tabular}{|c|c|c|c|c|c|}
\hline $\begin{array}{l}\text { Attractor } \\
\text { Series }\end{array}$ & $\begin{array}{c}\text { Fractal } \\
\text { Dimension } D_{0}\end{array}$ & $\begin{array}{c}\text { Information } \\
\text { Dimension } D_{1}\end{array}$ & $\begin{array}{c}\text { Correlation } \\
\text { Dimension } D_{2}\end{array}$ & $\begin{array}{c}\text { Lyapunov } \\
\text { Exponent } \lambda\end{array}$ & $\begin{array}{c}\text { Kolmogorov } \\
\text { Entropy }\end{array}$ \\
\hline Logistic pattern* & 1 & 0 & 0 & 0 & 0 \\
\hline$\Gamma$ & $>1$ & & & & .35 \\
\hline Logistic & .54 & & & .693 & .693 \\
\hline Baker & .57 & 1.572 & & 1 & 1 \\
\hline Henon 1.2 & $1.20,1.248$ & 1.283 & & & 1.20 \\
\hline Henon 1.4 & 1.26 & & 1.25 & .603 & 1.26 \\
\hline Zaslavskii & $1.39,1.792$ & 1.758 & $1.5,1.535$ & 3.689 & $<3.689$ \\
\hline Kaplan and Yorke & 1.43 & & 1.42 & & 1.43 \\
\hline Lorenz & $2.06,2.25$ & & 2.05 & 0.91 & 2.058 \\
\hline Random & & & & $>20 \dagger$ & $>10$ \\
\hline
\end{tabular}

There is some evidence (Gregson, 1988) that the brain involves at least two coupled attractors in processing sen-

Table 1

Estimates, Where Known, of Quantitative Measures of Chaotic Behavior for the Nine Systems Used

* Refers to the logistic map at parameter values that generate simple repetition and not chaotic dynamics.

$\dagger$ Refers to the largest exponent only. 
sory inputs. Two coupled strange attractors, with highdimensional noise superimposed, are necessary and sufficient (Arecchi \& Califano, 1987) to induce a $1 / \mathrm{f}$ distribution of frequencies (in which energy levels are inversely proportional to frequency) in observable output dynamics, which is what is seen in EEG activity. The Henon, Lorenz, Kaplan and Yorke (1979), Zaslavskii (1978), and $\Gamma$ cases, each built of two or more coupled recursions, might all implicate such two-attractor dynamics, or attractors that jump intermittently between two distinct patterns, and as such are all candidates, in principle, for mapping neuropsychophysical processes such as those underlying similarity judgments. The $\Gamma$ model is the only one for which there is evidence of appropriateness in modeling psychophysics, but only for modeling response levels and latencies, not for modeling similarities. The rest are not excluded precisely because they have not been explored in that context. The spectrum of $\Gamma$ is quite variable and not purely $1 / f$.

The question of interest is, do attractors that could represent features of psychophysical substrate activity also become the ones most discriminable from random noise? We think not necessarily so, but this is not the central issue of this experiment. The stimulus series used here constitute relatively slow-paced attractor dynamics that are fed into a system with much higher frequency chaotic behavior in its unstimulated states. The inputs are thus forcing functions transiently coupling two or more attractors over the duration of the auditory input series. Haken (1983) has written on systems in which one attractor drives another, in what is termed a slaving relationship; these systems exhibit complicated dynamics in many diverse phenomena in both physical and biological domains. The observed behavior in the current task is not simply a comparison of two attractors but rather a comparison of the separate results of their interactions with already existing brain dynamics. Knill, Field, and Kersten (1990) have recently offered evidence of optimal input processing in vision that is related to the fractal dimensionality of twodimensional displays, which suggests that the selective filtering of inputs is associated with some modality-specific chaotic mechanisms. Although the present experiment is encoded in sounds, the representation of individual frequencies in the peripheral receptors is a minor factor here and does not furnish the attractor dynamics of interest.

\section{METHOD}

\section{Design}

Harvey (1990) constructed the original experiment so that each series was 150 sounds long and took $30 \mathrm{sec}$ to play. For difference equations, the series were generated recursively. For the Lorenz equations, a fourth-order Runge-Kutta algorithm, with step size 0.025 and sampled at 1.1 times average rotational period, was used. For each attractor time series, the first 100 points were discarded to ensure that the process was on the attractor. The 150 points used were quantized linearly as integer values between 0 and 60 . Kaneko (1986) used only seven levels, which aliases the fine structure of the dynamics. The random series were generated by using a multiplicative recursive algorithm, yielding a rectangular distribution between 0 and 1 . The computer-generated sound series were recorded on a high quality tape and recorder for presentation to the subjects.

The encoding of the 61 variable levels was in pure tones that ranged from $61.74 \mathrm{~Hz}$ to $1975.53 \mathrm{~Hz}$ in equal log increment steps; the ratio of successive steps was approximately 1.05 . These steps equate to the keys on a piano keyboard, over six octaves. This resembles Pressing's (1988) approach to nonlinear maps in musical design.

Individual note duration was $0.16 \mathrm{sec}$, with internote rests of $0.04 \mathrm{sec}$. The sound sampling rate was $22 \mathrm{kHz}$, and the sampling resolution was 8 bits. Pilot tests showed that series of 150 sounds were sufficient to characterize the attractors. The interval between series in a trial was $5 \mathrm{sec}$, and the silent interval between trials was $20 \mathrm{sec}$. A sample sequence to show the range of the pitches of sounds used was played at the start of the experiment.

\section{Task Instructions}

A brief questionnaire on the musical interests of the subjects was completed first. The subjects were told that the task was to identify the random member of a pair of sound sequences-random was defined (Oxford English Dictionary) as "haphazard, without aim purpose or principle' - and a sequence of the pitches used was played to show their range, amplitude, and pacing.

On each trial, after each pair, the subjects made three responses in the following order:

1. They identified the sound sequence in the pair that they thought was random.

2. They expressed their confidence in their identification on a 5 -step rating scale.

3. They rated the similarity of the two sound sequences on a scale from " $0=$ drawn from two completely different processes" to " $100=$ drawn from one identical sort of process."

Note that in this last task the subjects were not asked to say if two sequences were identical.

No feedback on correctness was provided for Group 1 , but in the second variant of the task, done only by Group 2 , the correct answers to the identification of the random member in each of the first five pairs only were provided $15 \mathrm{sec}$ after each pair had been played. All of the subjects were debriefed at the end of the experiment.

\section{Stimulus Sequence}

The design, shown in Table 2 , is a mirror image of 10 pairs in order; the order within a pair is reversed in the second half of the design.

\section{Subjects}

Groups of students doing a second-year undergraduate course in experimental design were used; they performed the task as a group in natural hearing conditions from a single loudspeaker source. The first group, $n=65$, consisted of subjects of more mature ages, in the range of 25 to 55 years. The second group, $n=76$, with feedback

Table 2

Sequences of Attractor Pairs

\begin{tabular}{cll}
\hline Trial Nos. & Sequence 1 & \multicolumn{1}{c}{ Sequence 2 } \\
\hline 1,20 & Henon 1.2 & Random 1 \\
2,19 & Lorenz & Random 2 \\
3,18 & Random 3 & Kaplan and Yorke \\
4,17 & Logistic patter & Random 4 \\
5,16 & Random 5 & Baker transformation \\
6,15 & Logistic map & Random 6 \\
7,14 & Random 7 & Random 1 \\
8,13 & Random 8 & Zaslavskii \\
9,12 & Random 9 & Henon 1.4 \\
10,11 & Gregson & Random 10 \\
\hline
\end{tabular}


Table 3

Distribution Parameters of Similarity Ratings for the Random-Random Pairings, Trials 7 and 14

\begin{tabular}{ccccccc}
\hline Group & Trial & $M$ & $S D$ & Skew/SE & Kurt $/ S E$ & Mode \\
\hline 1 & 7 & 62.92 & 27.37 & -1.90 & -1.25 & 90 \\
1 & 14 & 50.38 & 24.61 & -0.09 & -1.17 & 60 \\
2 & 7 & 63.28 & 21.05 & -1.08 & -0.65 & 70 \\
2 & 14 & 60.16 & 22.57 & -2.28 & -0.60 & 80 \\
\hline
\end{tabular}

on the first five trials, was in a younger age range, typically about 19 to 25 years. None had had any instruction on chaotic dynamics.

\section{RESULTS}

The distribution of similarity ratings is the focus of this analysis; other results on discriminability from the present study and a previous study are given as contextual material only. The responses on Trials 7 and 14 constitute controls because those pairs are both of the random-random form. If performance were perfect, then discriminability in the specific sense elicited here would be zero and similarity responses would be at, or near to, the extreme of a 0-1 scale, at 1 , implying identity. Some evidence of learning during a session is provided by the changes in the shape parameters of the distributions, as shown in Table 3. The implication of these results is that the local control trial within a half of the experimental sequence, as opposed to a pooled result over the total sequence, should be used as a basis for evaluating discriminatory performance on the other trials.

Tables 4, 6, and 7 show results for Group 1, and Tables 5, 8, and 9 show results for Group 2 . In Tables 4 and 5, Trials 4 and 17 are those using the simple pattern series, which is readily seen as being very different from its random comparison series. It thus yields the lowest mean similarity ratings of any pair.

Table 6 shows the $\chi_{2}^{2}$ values for the KolmogorovSmirnov two-sample procedure for the total sample of 65 subjects in Group 1, the worst 16 subjects (total correct identifications of 10 or less), and the best 26 subjects (at least 14 correct identifications). The differences are marked but also vary from one trial to another, confirming that the successful subjects, in discriminating, are utilizing different stimulus properties in assessing attractor similarity and are not simply being more sensitive to the same sorts of dissimilarity.

The perception of similarities (or, implicitly, dissimilarities) between the two sequences in a pair sometimes leads to the correct identification of relative randomness; at other times, it does not. There are 20 similarity distributions to consider, ignoring the distinction between similarities with and without correctness of identification that is retained in Table 4. Table 5 is the corresponding pattern to Table 4 for Group 2, with feedback on the first five trials only; there is a tendency for the standard deviations of similarity responses associated with correct discrimination to be smaller, and there is also some diminution of confidence associated with wrong discriminations. The statistics in Table 6 (and the corresponding Table 8) are based on converting the frequency distributions of similarity ratings into 10-step category scales, since the similarity continuum typically has about three bits of information transmission capacity (Gregson, 1975). Then, comparing these category probability distributions for both correct and incorrect discriminations on each trial over all subjects yields the $D_{(2)}$ statistic of the Kolmogorov-Smirnov

Table 4

Means and Standard Deviations for Similarities and Confidences

for Group 1, Without Feedback $(n=65)$

\begin{tabular}{|c|c|c|c|c|c|c|}
\hline \multirow[b]{3}{*}{ Trial } & \multicolumn{4}{|c|}{ Similarities } & \multicolumn{2}{|c|}{ Confidences } \\
\hline & \multicolumn{2}{|c|}{$\begin{array}{c}\text { Correct } \\
\text { Identifications }\end{array}$} & \multicolumn{2}{|c|}{$\begin{array}{c}\text { Incorrect } \\
\text { Identifications }\end{array}$} & \multirow{2}{*}{$\begin{array}{c}\text { Mean } \\
\text { Correct } \\
\text { Identifications }\end{array}$} & \multirow{2}{*}{$\begin{array}{c}\text { Mean } \\
\text { Incorrect } \\
\text { Identifications }\end{array}$} \\
\hline & $M$ & $S D$ & $M$ & $S D$ & & \\
\hline 1 & 65.025 & 26.750 & 62.000 & 24.042 & 1.825 & 2.040 \\
\hline 2 & 58.395 & 26.707 & 67.272 & 16.428 & 1.744 & 1.727 \\
\hline 3 & 42.317 & 23.739 & 57.875 & 22.280 & 2.390 & 1.833 \\
\hline 4 & 13.396 & 20.804 & 49.166 & 27.827 & 3.264 & 2.750 \\
\hline 5 & 40.446 & 24.133 & 36.666 & 30.551 & 2.554 & 2.556 \\
\hline 6 & 55.122 & 26.307 & 49.791 & 28.302 & 2.024 & 2.250 \\
\hline 7 & & & 62.923 & 27.371 & 0.000 & 1.846 \\
\hline 8 & 55.833 & 24.566 & 50.000 & 23.453 & 1.929 & 1.609 \\
\hline 9 & 42.553 & 28.244 & 50.000 & 25.441 & 2.383 & 2.556 \\
\hline 10 & 34.020 & 25.553 & 40.714 & 23.820 & 2.824 & 2.643 \\
\hline 11 & 27.909 & 20.686 & 29.000 & 29.479 & 2.855 & 2.800 \\
\hline 12 & 48.415 & 26.970 & 49.583 & 23.889 & 2.073 & 1.542 \\
\hline 13 & 50.000 & 29.318 & 42.174 & 22.450 & 1.786 & 2.087 \\
\hline 14 & & & 50.385 & 24.610 & 0.000 & 1.846 \\
\hline 15 & 39.634 & 25.240 & 41.666 & 25.277 & 2.488 & 2.042 \\
\hline 16 & 50.116 & 28.109 & 45.682 & 24.041 & 2.279 & 1.591 \\
\hline 17 & 6.100 & 12.462 & 20.000 & 27.568 & 3.560 & 3.200 \\
\hline 18 & 53.250 & 26.518 & 61.200 & 24.137 & 2.075 & 1.600 \\
\hline 19 & 40.769 & 26.227 & 44.038 & 19.857 & 2.282 & 1.731 \\
\hline 20 & 43.333 & 25.604 & 37.000 & 24.311 & 2.422 & 2.200 \\
\hline
\end{tabular}


Table 5

Means and SDs for Similarities and Confidences for Group 2, With Feedback $(n=76)$

\begin{tabular}{|c|c|c|c|c|c|c|}
\hline \multirow[b]{3}{*}{ Trial } & \multicolumn{4}{|c|}{ Similarities } & \multicolumn{2}{|c|}{ Confidences } \\
\hline & \multicolumn{2}{|c|}{$\begin{array}{c}\text { Correct } \\
\text { Identifications }\end{array}$} & \multicolumn{2}{|c|}{$\begin{array}{c}\text { Incorrect } \\
\text { Identifications }\end{array}$} & \multirow{2}{*}{$\begin{array}{c}\text { Mean } \\
\text { Correct } \\
\text { Identifications }\end{array}$} & \multirow{2}{*}{$\begin{array}{c}\text { Mean } \\
\text { Incorrect } \\
\text { Identifications }\end{array}$} \\
\hline & $M$ & $S D$ & $\boldsymbol{M}$ & $S D$ & & \\
\hline 1 & 70.667 & 21.156 & 64.464 & 25.362 & 2.208 & 1.714 \\
\hline 2 & 60.659 & 23.965 & 63.719 & 20.149 & 1.591 & 1.406 \\
\hline 3 & 54.657 & 24.214 & 66.666 & 11.551 & 1.940 & 2.000 \\
\hline 4 & 15.937 & 20.327 & 26.250 & 29.377 & 3.250 & 2.583 \\
\hline 5 & 49.104 & 20.075 & 54.444 & 11.415 & 2.299 & 2.000 \\
\hline 6 & 58.961 & 20.963 & 59.208 & 20.100 & 1.788 & 1.458 \\
\hline 7 & & & 63.276 & 21.052 & 0.000 & 1.513 \\
\hline 8 & 65.289 & 22.308 & 58.774 & 20.182 & 1.422 & 0.903 \\
\hline 9 & 58.109 & 21.991 & 57.381 & 25.006 & 2.091 & 1.571 \\
\hline 10 & 42.078 & 24.873 & 56.250 & 23.553 & 2.687 & 1.583 \\
\hline 11 & 34.986 & 23.645 & 52.499 & 14.795 & 2.903 & 1.000 \\
\hline 12 & 64.400 & 21.432 & 55.423 & 23.222 & 1.120 & 1.500 \\
\hline 13 & 61.879 & 22.472 & 55.558 & 24.065 & 1.121 & 1.465 \\
\hline 14 & & & 60.158 & 22.570 & 0.000 & 1.487 \\
\hline 15 & 56.220 & 22.013 & 56.588 & 24.742 & 1.966 & 2.118 \\
\hline 16 & 56.073 & 22.461 & 65.904 & 19.192 & 1.836 & 1.238 \\
\hline 17 & 11.301 & 20.927 & 15.999 & 22.628 & 3.630 & 2.667 \\
\hline 18 & 60.454 & 20.416 & 54.625 & 22.830 & 1.705 & 1.219 \\
\hline 19 & 53.389 & 21.313 & 59.825 & 21.224 & 1.667 & 1.075 \\
\hline 20 & 56.963 & 22.436 & 58.363 & 15.562 & 2.444 & 1.955 \\
\hline
\end{tabular}

two-sample test. This furnishes information on the shifts (if any) in perceived similarity asociated with making a correct identification of the random member within a pair.

Although the best 26 subjects (defined according to their total correct identification scores over the 18 pertinent trials) do show some much bigger differences on, for example, Trials 4, 10, and 17, there are other trials, such as 2,13 , and 20 , in which the otherwise poor performers are superior.

The average probability of correct identification over the last 13 trials involving random-nonrandom comparisons is deduced from the number of correct identifications tabulated in Tables 6 and 8; the probabilities for Groups 1 and 2 were .68 and .70, respectively. This implies that feedback did not enhance average performance, but there are changes in the homogeneity of performance across the group members, as noted by comparing Tables 7 and 9 .

If there were a common basis of similarity perception over all the pairs (excepting 7 and 14), then we might expect to obtain a strong first principal component. This does not happen. It is not even the case that corresponding pairs (trials involving the same attractors) match up regularly; the asymmetry suggests a marked learning effect during the course of the trial sequence. This situation is displayed in Table 7 for Group 1, for which only the first four eigenvectors are given. In the analogous $\mathrm{Ta}$ ble 9 for Group 2, the correspondence between factor 3 in Table 7 and factor 2 in Table 9 is clear, but the remainder clearly fail to match.

In a previous study, Harvey (1990) conducted a series of detailed analyses of the data in Group 1 for each of the stimulus series. The analyses showed the following:
1. The distribution of total correct identification scores over the whole set of 18 was found to be different from a random (binomial) distribution.

2 . The probability of correct identification was significantly different $\left(\chi^{2}\right)$ from chance for every attractor, even for the Zaslavskii attractor (1978), whose phase portrait closely resembles randomness.

Table 6

Summary Statistics for Group 1 (With Subgroups)

\begin{tabular}{|c|c|c|c|c|c|}
\hline Mix & $\begin{array}{c}\text { No. } \\
\text { Correct } \\
\text { Out of } 65\end{array}$ & $\begin{array}{l}D_{(2)} \\
\operatorname{Max}\end{array}$ & Total* & Worst $16^{*}$ & Best $26^{*}$ \\
\hline 1 & 40 & .003 & 0.330 & 0.894 & 0.631 \\
\hline 2 & 43 & .023 & 2.416 & 4.364 & 2.437 \\
\hline 3 & 41 & .158 & 15.880 & 4.610 & 13.891 \\
\hline 4 & 53 & .565 & 66.007 & 9.818 & 41.595 \\
\hline 5 & 56 & .140 & 16.897 & 3.438 & 12.589 \\
\hline 6 & 41 & .029 & 2.919 & 3.938 & 3.414 \\
\hline 7 & 65 & & & & \\
\hline 8 & 42 & .022 & 2.279 & 1.333 & 4.719 \\
\hline 9 & 47 & .127 & 13.889 & 1.867 & 12.593 \\
\hline 10 & 51 & .172 & 19.666 & 3.000 & 14.302 \\
\hline 11 & 55 & .156 & 18.537 & 3.000 & 9.308 \\
\hline 12 & 41 & .010 & 0.987 & 2.012 & 1.180 \\
\hline 13 & 42 & .010 & 1.043 & 2.404 & 0.670 \\
\hline 14 & 65 & & & & \\
\hline 15 & 41 & .072 & 7.198 & 1.800 & 4.277 \\
\hline 16 & 43 & .008 & 0.789 & 0.741 & 1.666 \\
\hline 17 & 50 & .613 & 69.319 & 10.371 & 32.051 \\
\hline 18 & 40 & .007 & 0.677 & 0.371 & 1.399 \\
\hline 19 & 39 & .024 & 2.308 & 0.467 & 0.755 \\
\hline 20 & 45 & .017 & 1.795 & 4.528 & 0.727 \\
\hline
\end{tabular}

Note- $-D_{(2)}$ is the KS test statistic based on a maximum cumulative difference in two distributions, which is approximately distributed under $H_{0}$ as $\chi_{2}^{2}$ (Walsh, 1965). $\quad{ }^{*} \chi^{2}(2 \mathrm{df})$. 
Table 7

Rotated Factor Loadings (Princomp) for Group 1

\begin{tabular}{|c|c|c|c|c|}
\hline \multirow[b]{2}{*}{ Variable } & \multicolumn{4}{|c|}{ Factor } \\
\hline & 1 & 2 & 3 & 4 \\
\hline 1 & .203 & -.054 & .883 & -.024 \\
\hline 2 & .067 & .313 & .775 & -.080 \\
\hline 3 & -.014 & .433 & .521 & .253 \\
\hline 4 & .006 & -.007 & -.079 & .807 \\
\hline 5 & .049 & .497 & .286 & .553 \\
\hline 6 & .392 & -.022 & .511 & .412 \\
\hline 7 & .035 & .070 & .142 & .090 \\
\hline 8 & .164 & .111 & .207 & -.089 \\
\hline 9 & .020 & .243 & .160 & .476 \\
\hline 10 & .505 & .157 & .001 & .487 \\
\hline 11 & .596 & .306 & .267 & .380 \\
\hline 12 & .576 & .242 & .230 & .260 \\
\hline 13 & .195 & .743 & .088 & .125 \\
\hline 14 & .489 & .626 & -.044 & .089 \\
\hline 15 & .690 & .329 & -.007 & -.081 \\
\hline 16 & .077 & -.009 & -.019 & .175 \\
\hline 17 & .742 & .086 & .194 & -.125 \\
\hline 19 & .466 & .427 & .017 & .295 \\
\hline 20 & .493 & .231 & .068 & .182 \\
\hline VP & 2.991 & 2.563 & 2.228 & 2.091 \\
\hline
\end{tabular}

Note-VP is the eigenvalue (out of a total of 20).

3. The regression of mean confidence ratings on the probability of correct identification was

$$
\begin{gathered}
\text { Conf }=3.803 \cdot p(\text { correct })-.509, R^{2}=.765, \\
F=51.97, p<.001 .
\end{gathered}
$$

4. The mean confidence was greater when correct identification occurred for the pattern, $\Gamma$, Baker, Lorenz, Henon 1.4, Kaplan and Yorke (1979), and Zaslavskii (1978) attractors.

Tests on the actual sequential properties of the series of 150 sounds showed the following:

1. A departure from a rectangular probability distribution for all except the Baker attractor and, of course, random series (Kolmogorov-Smirnov one-sample tests).

2. The value of the peak frequency in a series is weakly positively correlated with correct identification.

3. The distribution of serial differences $\Delta_{y}^{1}$ is more unstable in chaotic than in random series; the $\Delta_{y}^{1}$ for a rectangular random series have a triangular distribution (as is known; Jeffreys, 1961).

4. In time series analyses, the chaotic series generally show significant AR1 coefficients and sometimes AR2 and AR3 coefficients; lack of these, however, does not prevent a series such as the logistic map from being discriminated from random series.

5. The unusually high coefficients (AR1 + AR2) for the Baker series, as opposed to the rest, provides a discrimination basis for this series that was otherwise unavailable, as it is not significantly different from a rectangular frequency distribution.

6. A Fourier analysis indicated that the $\Gamma$ and Henon series have high spectral components compared with the other series.

\section{DISCUSSION}

It is legitimate to conclude that no single linear loworder statistical measure is sufficient to serve as a common basis for the discriminability of the whole set of attractors. In the light of recent and powerful results (Green \& Savit, 1991; Savit \& Green, 1991), it is now known that it is not sufficient to compare human observer per-

Table 8

\begin{tabular}{|c|c|c|c|}
\hline \multicolumn{2}{|c|}{ No. Correct } & \multirow[b]{2}{*}{$D_{(2)} \operatorname{Max}$} & \multirow[b]{2}{*}{ Total* } \\
\hline Mix & Out of 76 & & \\
\hline 1 & 48 & .065 & 7.681 \\
\hline 2 & 44 & .010 & 1.099 \\
\hline 3 & 67 & .029 & 4.063 \\
\hline 4 & 64 & .550 & 76.467 \\
\hline 5 & 67 & .101 & 14.417 \\
\hline 6 & 52 & .013 & 1.587 \\
\hline 7 & 76 & & \\
\hline 8 & 45 & .004 & 0.494 \\
\hline 9 & 55 & .016 & 1.990 \\
\hline 10 & 64 & .181 & 25.121 \\
\hline 11 & 72 & .217 & 32.066 \\
\hline 12 & 50 & .023 & 2.714 \\
\hline 13 & 33 & .003 & 0.316 \\
\hline 14 & 76 & & \\
\hline 15 & 59 & .033 & 4.389 \\
\hline 16 & 55 & .025 & 3.250 \\
\hline 17 & 73 & .616 & 91.688 \\
\hline 18 & 44 & .005 & 0.518 \\
\hline 19 & 36 & .033 & 3.211 \\
\hline 20 & 54 & .007 & 0.836 \\
\hline
\end{tabular}

Summary Statistics for Group 2

Note- $D_{(2)}$ is the KS test statistic based on a maximum cumulative difference in two distributions, which is approximately distributed under $H_{0}$ as $\chi_{2}^{2}$ (Walsh, 1965). $\quad{ }^{*} \chi^{2}(2 \mathrm{df})$.

Table 9

Rotated Factor Loadings (Princomp) for Group 2

\begin{tabular}{ccccc}
\hline & \multicolumn{4}{c}{ Factor } \\
\cline { 2 - 5 } Variable & 1 & \multicolumn{1}{c}{2} & \multicolumn{1}{c}{3} & \multicolumn{1}{c}{4} \\
\hline 1 & .276 & .812 & .038 & .223 \\
2 & .231 & .819 & .174 & .280 \\
3 & .187 & .797 & .251 & -.054 \\
4 & .042 & .125 & .138 & -.141 \\
5 & .458 & .082 & -.088 & .502 \\
6 & .565 & .347 & -.148 & .130 \\
7 & .729 & .287 & .198 & .037 \\
8 & .673 & .258 & .265 & .188 \\
9 & .615 & .122 & .390 & .138 \\
10 & .659 & .030 & .291 & .132 \\
11 & .641 & .108 & .183 & .298 \\
12 & .380 & .089 & .338 & .532 \\
13 & .336 & .238 & .003 & .723 \\
14 & .603 & .223 & .162 & .440 \\
15 & .633 & .168 & .372 & .197 \\
16 & .310 & .169 & .697 & .160 \\
17 & .036 & .120 & -.038 & .050 \\
18 & .223 & -.070 & .650 & .456 \\
19 & .015 & .167 & .503 & .699 \\
20 & .224 & .284 & .640 & -.049 \\
VP & 4.123 & 2.585 & 2.375 & 2.347 \\
\hline
\end{tabular}

Note-VP is the eigenvalue (out of a total of 20). 
formance in assessing time series similarities by employing the linear statistics used in computing autocorrelations and partial autocorrelations; because those linear statistics can fail to detect the deterministic nonlinear dynamics that distinguish a random independent identically distributed (i.i.d.) stochastic process from the evolution of low-dimensional attractors. This implies that the human observer need not use (and cannot necessarily be postulated to use) some analog of autoregression estimation to assess the similarities between the finite realizations used here.

As Green and Savit (1991, p. 525) observe, "it may happen that the autocorrelation functions of a time series are zero, while the elements of the series have nontrivial statistical interdependencies," and, again (Savit \& Green, 1991, p. 97), "many sequences generated by deterministic maps pass standard tests of randomness based on autocorrelation functions.' Analogously to some of the measures used heuristically by Harvey (1990) previously, Savit and Green (1991) suggest that predictability can be defined as a function of the probability of reproducible subsequences being observed; this recurrence of subsequences can be conceived as an intermediate stage between complete randomness in the i.i.d. sense and complete predictability. This idea can be used to furnish a basis for similarity judgments on long time series samples; as the proportion of the series that appears to the observer to be composed of little runs of a recognizable structure increases, its similarity to randomness decreases, provided that such runs are recognized as such. Clearly, one could have hits and misses in that recognition task itself. This hypothesis makes no assumptions about the Gaussian or non-Gaussian form of the i.i.d. series and can cover cases in which the series is generated from a mixture of deterministic and stochastic epochs.

The relative discriminability of the attractors can be expressed in various ways: the probability of identification, the associated chi-square in the Kolmogorov-Smirnov twosample test on the similarity distributions, and the analog of $d^{\prime}$ derived from the plots resembling receiver-operating characteristics (ROC), as shown in Figures 1 and 2 for Group 1, without feedback, and Figures 3 and 4 for Group 2, with feedback. These are analogs of ROC plots and are innovative in the sense that they are based on similarity distributions, which span the $0-1$ interval, and not on probabilities that are on the same interval but also satisfy axioms not defined on similarity measures.

It is expected that there should be a relationship between Kolmogorov-Smirnov statistics and the sense of $d^{\prime}$ from their derivation (Durbin, 1971). Figure 3 is not strictly interpretable because of the feedback on five of the nine trials plotted. The use of the normal deviate transform of probabilities (Johnson \& Kotz, 1970) should give a set of parallels to the line with unit slope in the $z-z$ plane if the process is the standard signal detection theory model with two Gaussian distributions on the decision axis. It does not; Figures 5 and 6 show a convergent pattern similar to a fan.
Some interpretation is clear-the clustering of like trials $(1,20 ; 2,19 ; 3,18$, etc. $)$ shows weakly. This figure should be compared with Table 2 for interpretation.

In Group 2, the feedback appears to induce more homogeneity in performance, but a secondary mode of poorer performers still exists. The increase in the first eigenvalue (compare Tables 7 and 9) also confirms the greater induced homogeneity in Group 2.

These methods all show some mutual agreement and confirm that some attractors are readily discriminable from random sequences of the types used here, whereas others are not.

The logistic pattern $(4,17)$ is most clearly not perceived as random, followed by the Gregson $\Gamma$ process $(10,11)$, and then by Henon (9) or Baker (5). The fact that the similarity distributions are multimodal suggests that the relationship between perceived similarity and the identification of an attractor sequence as nonrandom is a complicated process and is heterogenous over a sample of subjects. The question of interest is, are there mathematically definable properties of these samples that characterize them in a way that relates to the results in Figures 1-6? Some of them show strong quasiperiodicities and arpeggio-like subsequences. The dimensionality of most of the random processes used here, which were computer generated, is integer, but all of the chaotic attractors have fractal dimensions; the discrimination could interestingly, therefore, be one between fractal and integer dimensionality. So, from a cognitive approach, one might view the problem as both a detection process that stochastically recognizes local recurrent subsequences and a decision process post hoc that accepts or rejects them as possible within a random series of sufficient length. It is not a settled question concerning what subjects actually think randomness looks like in series, and individual differences in the tolerance of fortuitous local apparent structure in series could be a source of the variability in Tables 4 and 5 . Although some of the chaotic series pass traditional tests for randomness, the human observer, with finite pitch-resolution capacity and an ability to form similarity perceptual relationships, may apparently detect underlying structural properties that escape linear statistical tests.

Any stationary finite series can be characterized to an arbitrary degree of precision by a moving-average autoregressive process, and so the autoregressive spectrum of some of these samples is a starting point for the examination of the presence of detectable subsequences with quasiperiodic structure. Fitting a purely autoregressive model to the $y$ evolution in Equation 10, for example, gives components for $a=4.27, e=.06$, of lag values for autoregressions of $1,2,3,5,10,18,20$, whereas a very small shift to $a=4.25, e=.05$ gives only autoregressive lags of $1,2,20$. In both cases, a large residual at lag 20 still persists. The values used are in the neighborhood of an attractor. The variability in local autoregression indicates that even one of the attractor equations used here could be the source of a diversity of sequences with different dis- 
Probability distributions of Similarities

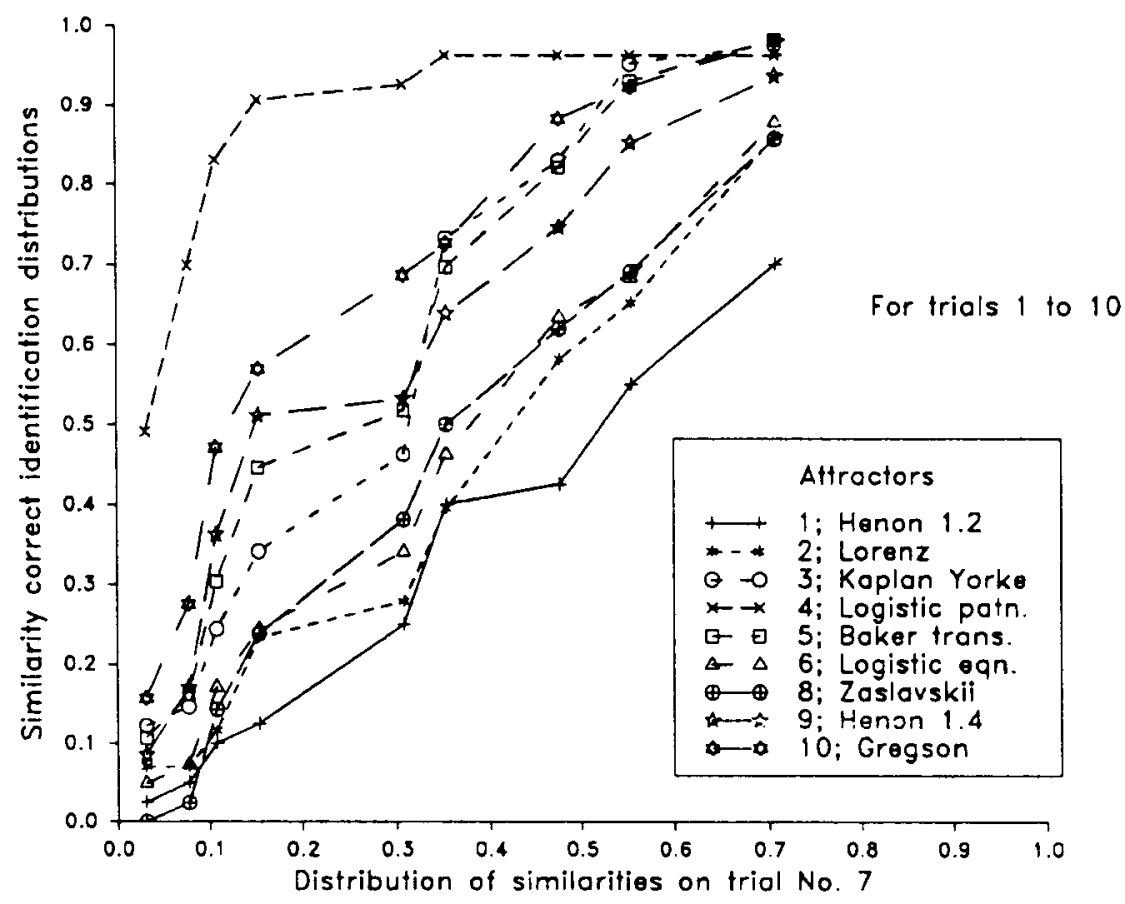

Figure 1. Similarity analogs of ROC curves, Trials 1-10.

Probability distributions of Similarities

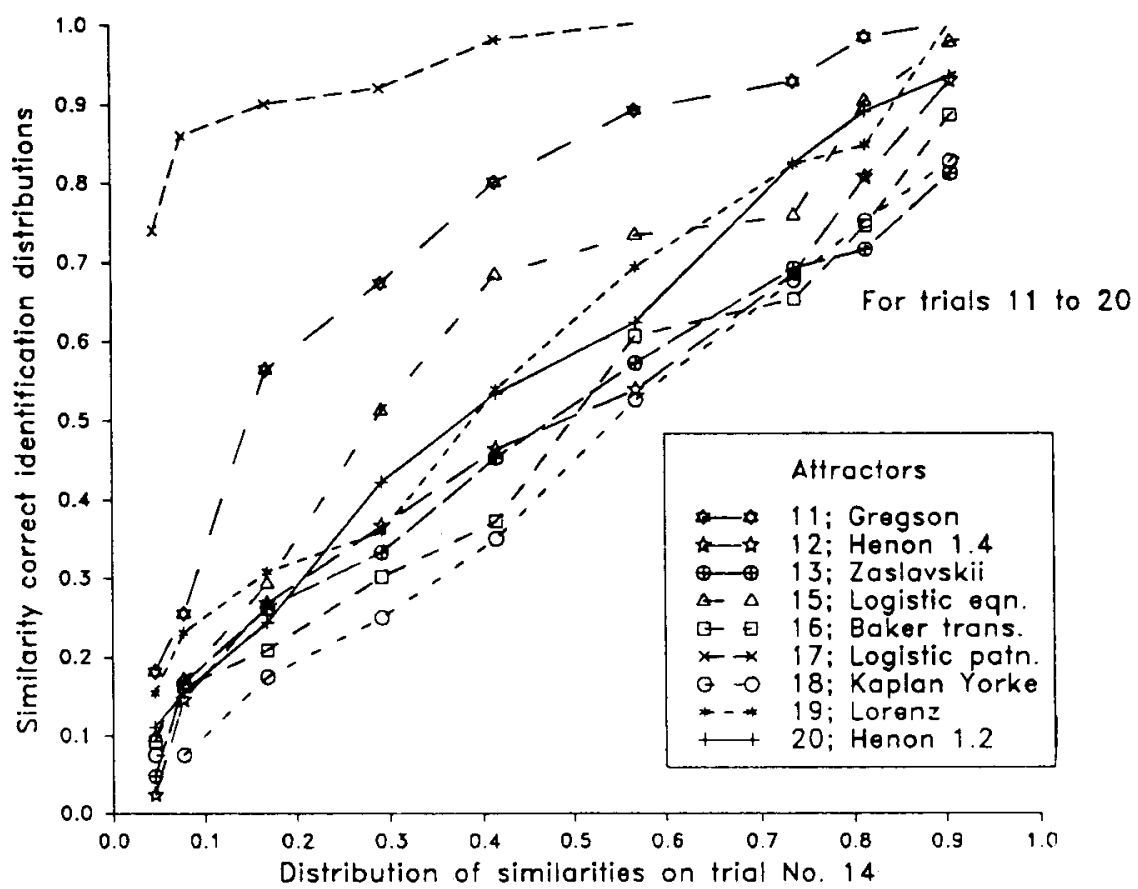

Figure 2. Similarity analogs of $\mathrm{ROC}$ curves, Trials 11-20. 
Probability distributions of Similarities

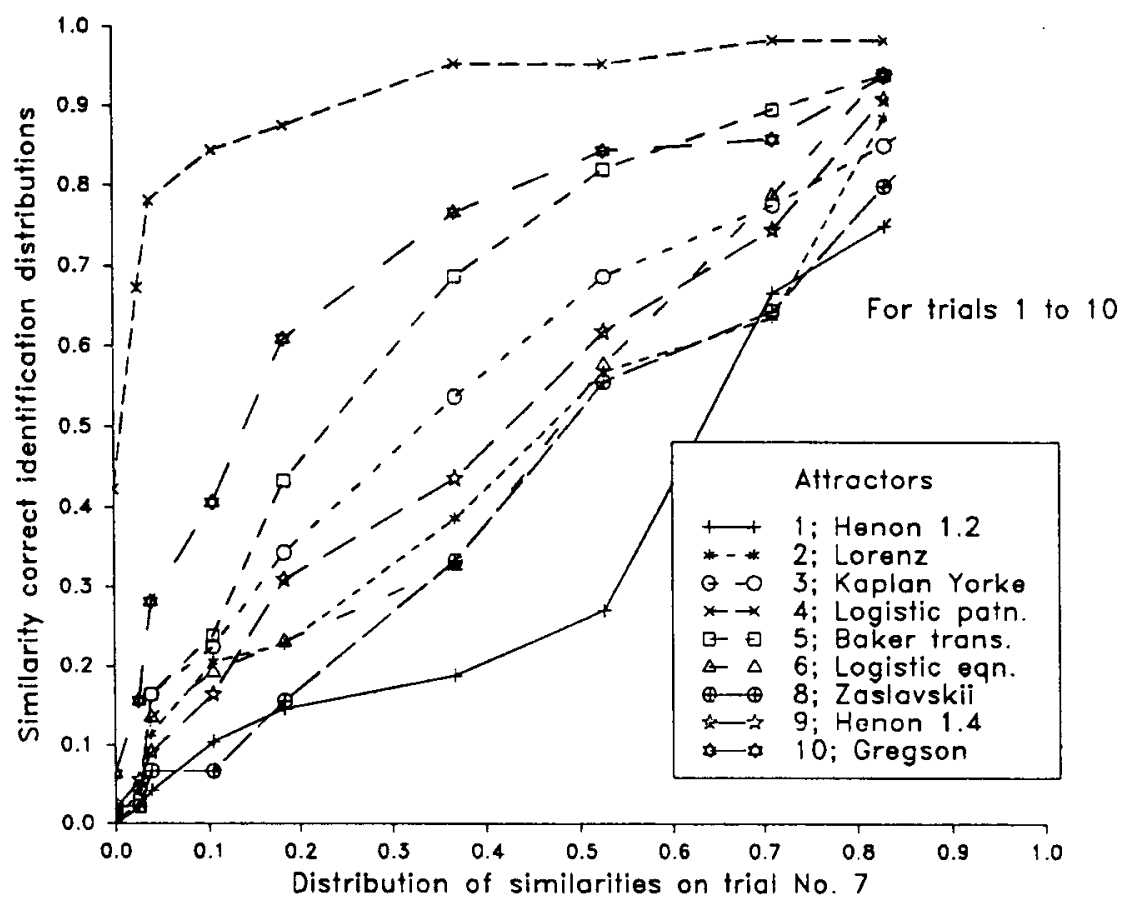

Figure 3. As in Figure 1, but with feedback in the second group.

Probability distributions of Similarities

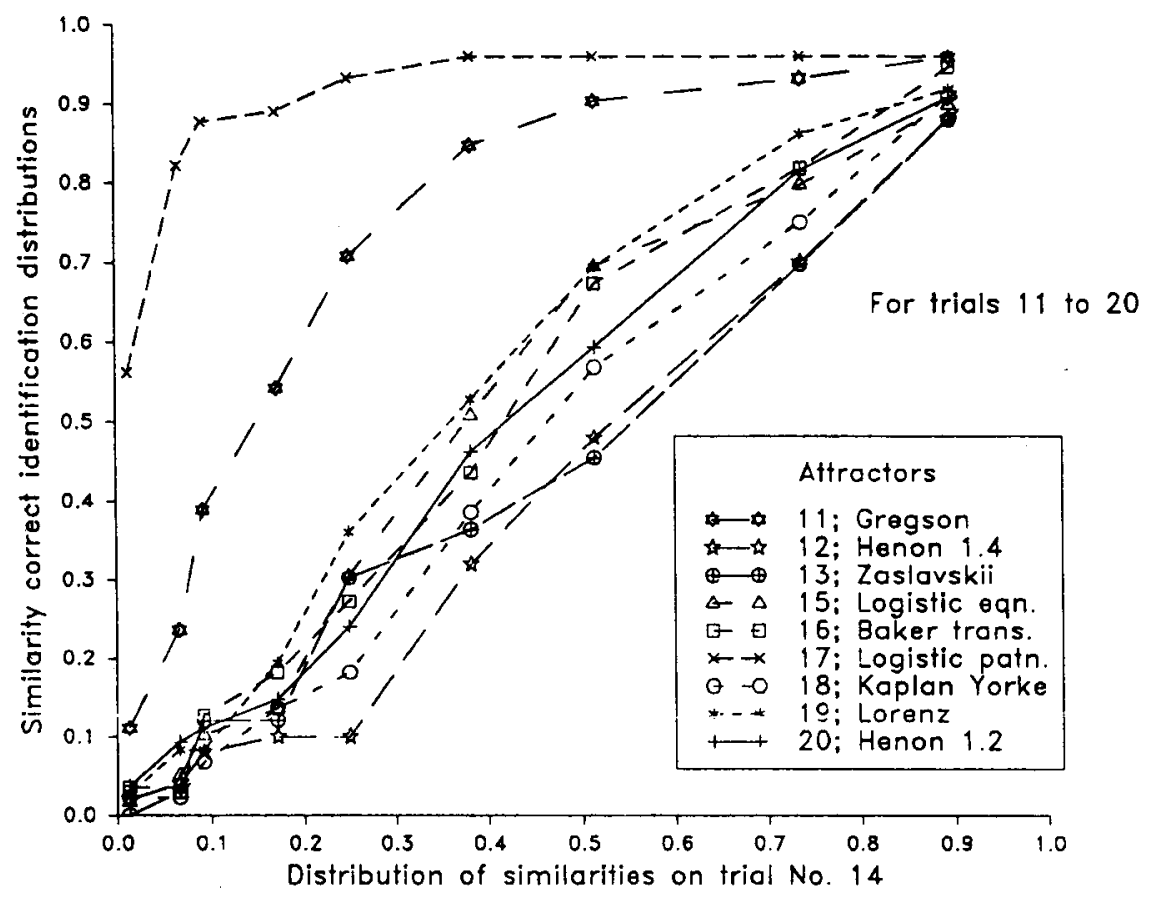

Figure 4. As in Figure 2, but with feedback in the second group. 
Normal deviate transforms of Similarity R.O.C.S

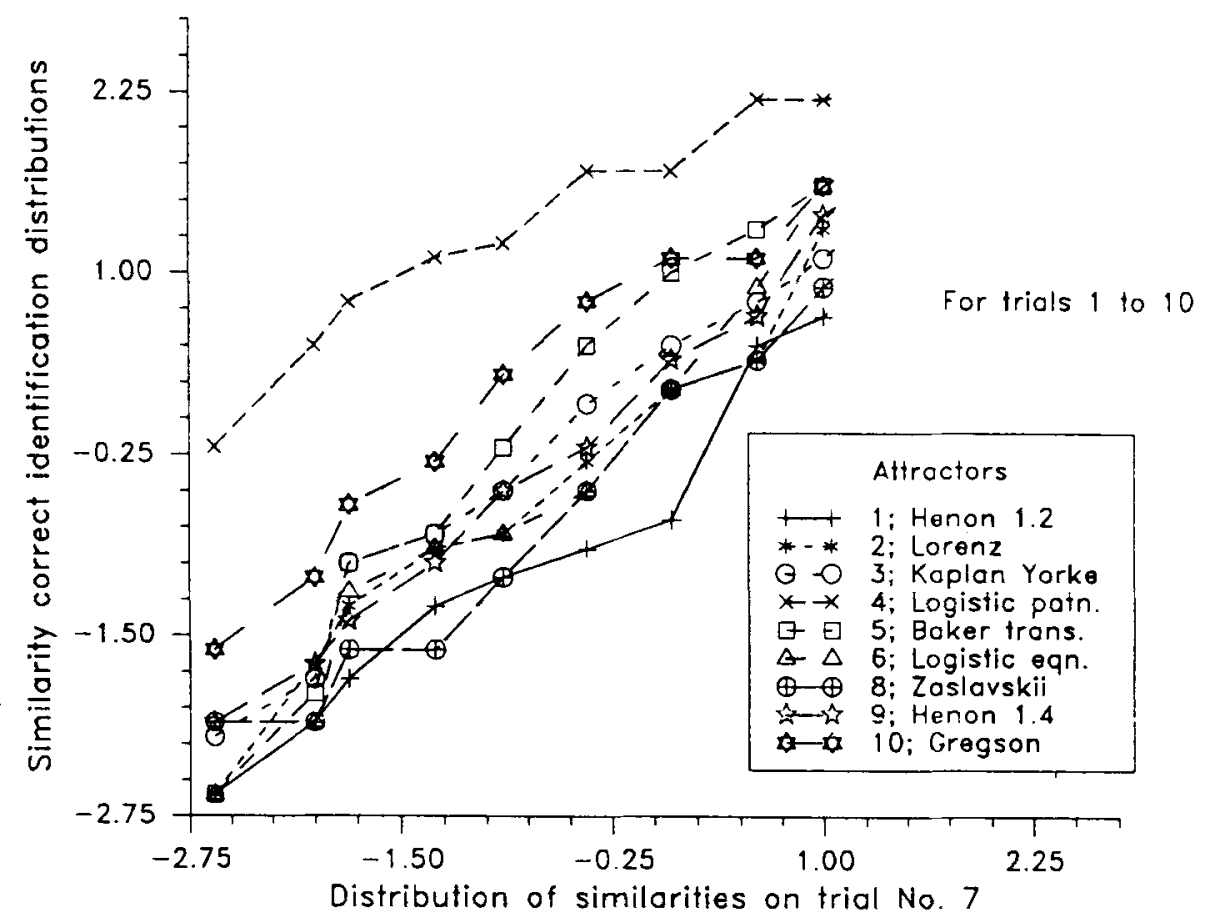

Figure 5. Transformation of Figure 1 into $z$ scores.

Normal deviate transform of Similarity R.O.C.S

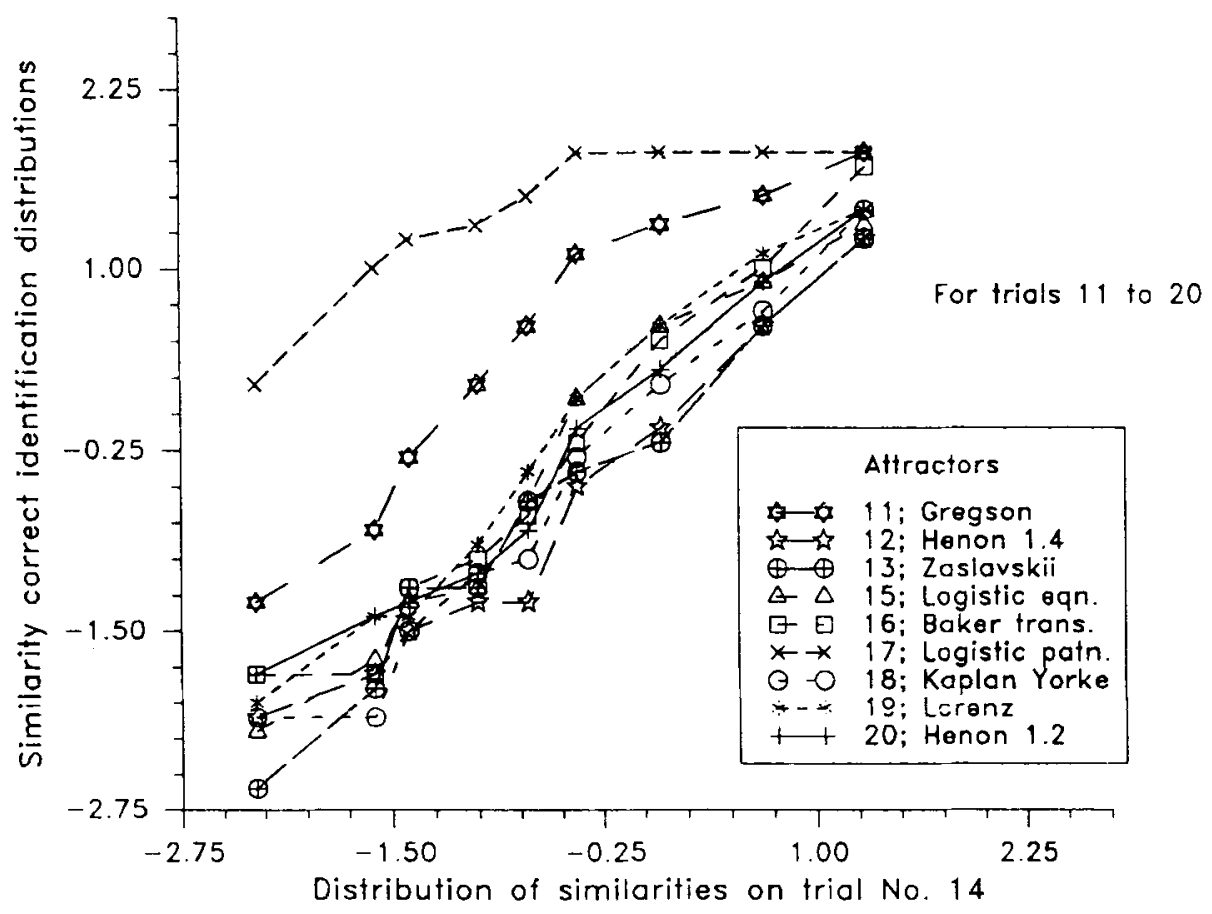

Figure 6. Transformation of Figure 2 into $z$ scores. 
criminabilities from random series. Hence, the experiment is better considered as one of perceived similarity between sampled realizations from a set of families of attractors rather than as a comparison of the nine attractors themselves, each generating its unique form; there is not necessarily a unique chaotic pattern unless the parameters are fixed, because as soon as a Devil's Staircase is formed, a set of points that exhibit incommensurate dynamics can arise.

\section{REFERENCES}

Arecchi, F. T., \& Califano, A. (1987). Noise-induced trapping at the boundary between two attractors: A source of $1 / \mathrm{f}$ spectra in nonlinear dynamics. Europhysics Letters, 3, 5-10.

Box, G. E. P., Jenkins, G. M. (1970). Time series analysis, forecasting and control. San Francisco: Holden-Day.

BRIGGs, K. M. (1990). Improved methods for the analysis of chaotic time series (Mathematics Research Paper, No. 90-2). Melbourne, Australia: La Trobe University, Department of Mathematics.

Campbell, E. A., \& Gregson, R. A. M. (1990). Julia sets for the gamma recursion in nonlinear psychophysics. Acta Applicandae Mathematicae, 20, 177-188.

Durbin, J. (1971). Boundary-crossing probabilities for the Brownian motion and Poisson processes and techniques for computing the power of the Kolmogorov-Smirnov Test. Journal of Applied Probability, 8 , 431-453.

Feder, J. (1988). Fractals. New York: Plenum.

GreEN, M. L., SAVIT, M. (1991). Dependent variables in broad based continuous time series. Physica, 50D, 521-544.

Gregson, R. A. M. (1975). Psychometrics of similarity. New York: Academic Press.

Gregson, R. A. M. (1983). Time series in psychology. Hillsdale, NJ: Erlbaum.

GrEGSON, R. A. M. (1988). Nonlinear psychophysical dynamics. Hillsdale, NJ: Erlbaum.

GREGSON, R. A. M. (1989a). Nonlinear psychophysics in higher dimensionality. Paper presented at the 20th meeting of the European Mathematical Psychology Society, Catholic University of Nijmegen, Netherlands.

Gregson, R. A. M. (1989b). A nonlinear systems approach to Fechner's paradox. Biological Cybernetics, 61, 129-138.

Gregson, R. A. M., \& Britton, L. A. (1989). The size-weight illusion in 2-D nonlinear psychophysics. Perception \& Psychophysics, 48, 343-356.
Gumowski, I., \& MirA, C. (1980). Dynamique chaotique. Toulouse, France: Cepadues Éditions.

HAKEN, H. (1983). Advanced synergetics. Berlin: Springer-Verlag.

HARVEY, J. P. (1990). Auditory detection of chaos. Unpublished master's thesis, University of New England, New South Wales, Australia.

JEFFREYs, H. (1961). Theory of probability (3rd ed.). Oxford: Clarendon Press.

Johnson, N. L., Kotz, S. (1970). Continuous univariate distributions -1. New York: Houghton Mifflin.

KANEKo, K. (1986). Collapse of tori and genesis of chaos in dissipative systems. Singapore: World Scientific.

Kaplan, J. L., \& YoRKE, J. A. (1979). Chaotic behavior of multidimensional difference equations. In H.-O. Peitgen \& $\mathrm{H}$.-O. Walther (Eds.), Lecture notes in mathematics (Vol. 730, pp. 228-234).

KNill, D. C., Field, D., Kersten, D. (1990). Human discrimination of fractal images. Journal of the Optical Society of America, A, 7, 1113-1123.

MANDELBROT, B. (1977). Fractals: Form, chance and dimension. San Francisco: W. H. Freeman.

Pressing, J. (1988). Nonlinear maps as generators of musical design. Computer Music Journal, 12, 75-88.

Price, I. R., \& Gregson, R. A. M. (1988). Nonlinear dynamics in a complex cubic one-dimensional model for sensory psychophysics. Acta Mathematicae Applicandae, 11, 1-17.

SAvit, M., GReEN, M. L. (1991). Time series and dependent variables. Physica, 50D, 95-116.

WALSH, J. E. (1965). Handbook of nonparametric statistics II. Princeton: Van Nostrand.

Wolf, A., SWift, J. B., SWinney, H. L., \& Vastano, J. A. (1985). Determining Lyapunov exponents from a time series. Physica, 16D, 285-293.

ZASLAVSKII, G. M. (1978). The simplest case of a strange attractor. Physics Letters, 69A, 145-147.

\section{NOTES}

1. The Zaslavskii (1978) map is known to be mathematically aberrant, in the sense of failing to satisfy some criteria that the Henon and some other equations are known to meet.

2. This problem seems to have been noticed first by Mandelbrot (1977).

(Manuscript received January 2, 1991; revision accepted for publication September 27, 1991.) 